\title{
Indices for Assessment of the Quality of Glycemic Control and Glucose Dynamics from Continuous Glucose Monitoring
}

\author{
Klaus-Dieter Kohnert ${ }^{1 *}$, Peter Heinke ${ }^{1}$, Lutz Vogt ${ }^{2}$, Petra Augstein ${ }^{1,3}$, Andreas Thomas $^{4}$ and Eckhard \\ Salzsieder ${ }^{1}$
}

${ }^{1}$ Institute of Diabetes "Gerhardt Katsch" Karlsburg, Germany

${ }^{2}$ Diabetes Service Center, Karlsburg, Germany

${ }^{3}$ Department of Medicine A, University Medicine Greifswald, Greifswald, Germany

${ }^{4}$ Medtronic $\mathrm{GmbH}$, Meerbusch, Germany

*Corresponding author: Klaus-Dieter Kohnert, M.D, Ph.D., Institute of Diabetes "Gerhardt Katsch" Karlsburg, Germany, Tel: +49-0-3835568406, Fax: +49-0-3835568444, E-mail: kohnert@diabetes-karlsburg.de

\begin{abstract}
Objective: To compare indices of the quality of glycemic control and their association with glucose complexity, using composite measures of glycemia and multiscale entropy derived from continuous glucose monitoring datasets.

Study design: A retrospective cohort study.

Methods: Continuous glucose monitoring data obtained during an observational study involved 256 subjects with type 1 $(n=22)$, type 2 diabetes $(n=222)$, and 12 non-diabetic control subjects. We compared the indices for the quality of glycemic control and determined their relationship with glucose profile complexity. The indices evaluated were: Low Blood Glucose Index, High Blood Glucose Index, Average Daily Risk Range (ADRR), Glycemic Risk Assessment Diabetes Equation (GRADE) scores, and the recently introduced Q-score. We used multiscale entropy analysis of the datasets to assess the relationship between glucose dynamics and the indices for the quality of glycemic control.

Results: We categorized the study cohort into four groups according to the Q-score ( $\leq 5.9$; Good, 6.0-8.4; Satisfactory, 8.5-11.9; Fair and $\geq 12.0$; Poor). An increase in the Q-score was related with worsening of glycemic control as demonstrated by progressively elevated levels of the glucose exposure metrics $\mathrm{HbA} 1 \mathrm{c}$, mean glucose, and the glycemic variability measure \%CV. In contrast, the multiscale entropy index decreased across the quality categories, attaining its lowest value at the highest Q-score regarded as Poor. ADRR, GRADE, and Q-score were highly correlated to one another and positively associated with the antidiabetes therapy ( $r=0.424$ to 0.469$)$, but inversely correlated with the multiscale entropy $(r=-0.337, p<0.001$; $-0.286, p<0.001 ;-0.353, p<0.001)$.
\end{abstract}

Conclusions: Indices assessing the quality of glycemic control yield a more comprehensive picture of glucose profiles than just defining a pre-specified target range or the mean value and its dispersion. The multiscale entropy complements these indices by providing insights into the dynamics of the glucoregulatory system not otherwise accessible.

\section{Keywords}

Indices of quality of glycemic control, Multiscale entropy, Antidiabetes therapy, Type 1 and Type 2 diabetes

\begin{abstract}
Abbreviations
ADRR = Average Daily Risk Range; GRADE = Glycemic Risk Assessment Diabetes Equation; GRADE hypo $=$ GRADE hypoglycemia (<3.9 mmol/L); GRADE $_{\text {eu }}=$ GRADE euglycemia $(\geq 3.9-\leq 8.9 \mathrm{mmol} / \mathrm{L}) ; \mathrm{GRADE}_{\text {hyper }}=\mathrm{GRADE}$ hyperglycemia (> $8.9 \mathrm{mmol} / \mathrm{L})$; MSE = Multiscale Entropy; Q-score = Quality Score of Glycemic Control; SampEn = Sample Entropy
\end{abstract}

\section{Introduction}

Blood glucose control is a fundamental element in the management of diabetes to reduce or even prevent micro- and macrovascular complications [1]. Many patients with diabetes can improve glycemic control by use of Continuous Glucose Monitoring (CGM) systems. However, analysis and interpretation of the datasets obtained from CGM are complicated by the many indices and measures currently proposed for assessment of 
glycemic variability and the quality of glycemic control [2]. One must consider that measures of the quality of glycemia are not interchangeable with indices of glycemic variability because they reflect different aspects of diabetes control. Furthermore, for practical reasons, it might be advantageous to integrate multiple parameters of glycemic control, e.g., hypo-, hyperglycemia, and glycemic variability, into one single index or score to assess at a glance the metabolic state of the patient and the efficiency of therapeutic intervention.

Kovatchev, et al. [3] introduced the Average Daily Risk Range (ADRR) with the Low (LBGI) and High Blood Glucose Index (HBGI), and Hill, et al. [4] developed the Glycemic Risk in Diabetes Equation (GRADE) with \%GRA-

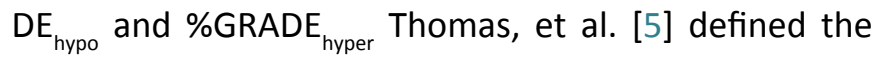
Glucose Pentagon, which summarizes measures characterizing glucose variability and long-term glycemic control. Rodbard $[6,7]$ used stacked bar charts for display of glucose distributions and quartiles for the percentage coefficient of variation $(\% \mathrm{CV})$ to compare the quality categories of glycemic control between patient groups. And we have recently presented the Q-score as a novel metric enabling therapy stratification of diabetic patients [8]. We further noted that the Q-score allowed categorization of CGM profiles ranging from excellent to poor and showed that the scores varied with therapy escalation.

Beyond estimates of the quality of glycemic control, measures such as Multiscale Entropy (MSE), characterizing the dynamics of glucose time series have gained increasing attention. The MSE approach, introduced by Costa, et al. [9], has the potential to assess how treatment modalities can modify the dynamics of glucose regulation. Thus, quality scores and dynamical measures of glucose time series could provide adequate means to target glycemic control and the glucoregulatory system. However, comparisons regarding the utility of different quality scores lack as yet. And the relationships with the dynamics of glucose profiles are unknown.

Therefore, the aim of our study was to compare various indices of the quality of glycemic control derived from CGM datasets and their relationship with the dynamics of the glucose profiles.

\section{Subjects and Methods}

\section{Database}

The present investigation is cross-sectional and used anonymized data derived from retrospective ambulatory CGM profiles (MiniMed Solution Software, Medtronic MiniMed) obtained on our own patients and non-diabetic control subjects. The patients had been referred from primary care practices distributed through Germany and entered without any selection. Non-diabetic control subjects were included to cover the whole spectrum of glycemia from normal to poor control. We applied established measures of glycemic control and glucose profile dynamics for the analysis of the CGM data obtained. Glucose sensors were placed on the patient's abdomen. Recordings with a mean duration of 60-h and a minimum of four glucose meter calibrations per day provided the data for the calculations. We strictly obeyed the validity criteria of the manufacturer $(\geq$ 3 paired sensor/meter readings and mean absolute difference $\leq 28 \%$ ).

\section{Study subjects}

Fourty-two of the study subjects had diet alone. Of the 180 patients with Type 2 Diabetes (T2D) assigned to oral drugs, 34 had received metformin, 37 sulfonylurea, four glitazones, 11 dipeptidyl peptidase-4 inhibitors alone, and 34 combinations thereof. Thirty-five patients had insulin plus oral anti diabetes agents, and 25 had insulin alone. Twenty-two patients with Type 1 Diabetes (T1D) had received multiple insulin injections consisting of short- and long-acting insulin. Seventy-four percent of the patients with diabetes had taken blood pressure lowering medication. Since the original study [10] had received ethical approval and, before inclusion, all study participants provided their written informed consent, this retrospective data analysis required no further approval.

\section{Indices of quality of glycemic control}

These indices included the Low Blood Glucose Index (LBGI), High Blood Glucose Index (HBGI), Average Daily Risk Range (ADRR) [3], Glycemic Risk Assessment Diabetes Equation (GRADE), \%GRADE ${ }_{\text {hypo }}, \% G R A D E_{\text {eu' }}$ \%GRA$\mathrm{DE}_{\text {hyper }}$ [4], and the recently introduced Q-score [8]. The $\mathrm{Q}$-score is based on factor analysis, using z- scores and combines mean glucose, glucose range, time in glycemia $\left(\mathrm{t}_{\mathrm{G}}\right)<3.9 \mathrm{~mol} / \mathrm{L}$, and $>8.9 \mathrm{mmol} / \mathrm{L}$, and Mean of Daily Differences (MODD). The Q-score was computed according to the formula [8], as previously described:

$Q$-Score $=8+\frac{\text { mean glucose }-7.8}{1.7}+\frac{\text { range }-7.5}{2.9}+\frac{3.9-0.6}{2.9}+\frac{>8.9-6.2}{5.7}+\frac{M O D D}{0.9} 1.8$

The glycemic ranges defined as $<3.9 \mathrm{mmol} / \mathrm{L},>3.9$ to $<8.9 \mathrm{mmol} / \mathrm{L}$, and $>8.9 \mathrm{mmol} / \mathrm{l}$ were termed hypoglycemic, euglycemic, and hyperglycemic, respectively. Glycated Hemoglobin (HbA1c), used as glucose exposure measure, was determined by standard methods.

\section{Multiscale entropy analysis}

We analyzed the Multiscale Entropy (MSE) for the CGM sequences according to the SampEn algorithm previously described by Chen, et al. [11] and Costa, et al. [12]. In brief, this procedure comprised: (1) Derivation of a set of time series from the original glucose signal on different time scales using the coarse-graining technique, (2) Computation of SampEn with standard parameter values for each coarse-grained time series, choosing the window length $m=2$, the sensitivity criterion $r=$ 0.15 times the standard deviation, and the data length 
$\mathrm{N}=288$ within the entire coarse-grained sequence with the largest scale factor set at $M=7$, and (3) Plotting each calculated SamEn for the corresponding scale factor to obtain the MSE index as the sum over the range 1 to 7 .

\section{Statistical analysis}

We categorized the patients by their Q-score values. As described in detail elsewhere, three diabetes specialists evaluated the CGM profiles and provided the categorization according to the quality of glycemic control [8]. For comparison of continuous variables, we used either one-way Analysis of Variance (ANOVA) or Kruskal-Wallis one way analysis, as appropriate. Control of multiple pair wise comparisons was performed using the Holm-Sidak and Dunn's method, respectively. Spearman's correlation analysis and multivariate regression models were used to relate MSE complexity to indices of the quality of glycemic control and anti-diabetic therapy. Therapy was coded: 1 = non-diabetes, 2 = diet with/without oral anti-diabetic agents, $3=$ insulin with/without oral anti-diabetic agents, 4 $=$ T1D. Potential confounding variables were age, sex, diabetes duration, and body mass index. We used the Wald test, and variance inflation factor and Durbin-Watson statistic served as the criterion for collinearity. $\mathrm{P}<0.05$ was considered statistically significant. Statistical analysis used the Statistical Package for the Social Sciences software package (version 17.0; SPSS, Chicago, IL).

\section{Results}

Table 1 shows that the study subjects categorized according to their Q-scores were significantly older in the two lower than those in the two higher Q-score categories. Diabetes duration, $\mathrm{HbA1c}$, mean glucose, and \%CV increased across the groups, and all were greater in the highest as compared to the lower Q-score categories. However, BMI was not significantly different between the Q-score categories. These data indicate that high $\mathrm{Q}$-score values are related to worsening of glycemic control.

Levels for ADRR, GRADE, and the MSE index were associated with the quality of glycemic control. Figure 1 displays that ADRR and GRADE increased concomitant with the Q-score categories and were highest in the state of glycemia defined as poor. In contrast, the MSE index declined consistently across the quality categories from good to poor, demonstrating that worsening of diabetes control was associated with decreasing glucose profile complexity.

The degree of correlation between the quality scores was high (Table 2). Likewise, the relationship was strong with $\mathrm{HBGI}, \% \mathrm{GRADE}_{\text {hyper }^{\prime}}$ and \%GRADE ${ }_{\text {eu }}$, whereas the associations with hypoglycemia indices such as LBGI and $\% \mathrm{GRADE}_{\text {hypo }}$ were weak.

Table 1: Demographic and metabolic characteristics of study subjects by Q-score categories.

\begin{tabular}{|c|c|c|c|c|c|}
\hline \multirow[t]{2}{*}{ Characteristics } & \multicolumn{4}{|c|}{ Q-score category } & \multirow[t]{2}{*}{$P$ value } \\
\hline & Good $\leq 5.9$ & Fair 6.0-8.4 & Satisfactory 8.5-11.9 & Poor $\geq 12.0$ & \\
\hline Study subjects $(n)$ & 83 & 63 & 59 & 51 & \\
\hline Sex (male/female) & $49 / 34$ & $32 / 31$ & $32 / 27$ & $22 / 29$ & 0.34 \\
\hline Age (years) & $66.0(59.0-70)$ & $66.0(57.3-69.8)$ & $61.0(54.8-68.0)^{*}$ & $59.0(46.0-67.0)^{*}$ & 0.023 \\
\hline Diabetes duration (years) & $5.9(2.0-10.0)$ & $8.0(3.8-12.3)$ & $8.0(5.8-15.0)^{*}$ & $12.0(7.0-19.0)^{\star}$ & $<0.001$ \\
\hline Body mass index $\left(\mathrm{kg} / \mathrm{m}^{2}\right)$ & $29.4(26.5-32.1)$ & $30.1(27.8-32.3)$ & $30.8(28.1-35.8)$ & $30.0(25.5-34.3)$ & 0.25 \\
\hline Hemoglobin A1c (\%) & $6.3(5.7-6.9)$ & $6.5(5.8-7.1)$ & $7.2(6.5-7.6)^{\star} \dagger$ & $7.8(7.1-8.7)^{\star} \dagger$ & $<0.001$ \\
\hline$(\mathrm{mmol} / \mathrm{mol})$ & $42(39-52)$ & $48(40-54)$ & $55(48-60)$ & $62(54-72)$ & \\
\hline Mean glucose (mmol/L) & $6.5(6.0-7.0)$ & $7.3(6.8-8.0)^{*}$ & $8.6(7.7-9.3)^{\star} \dagger$ & $10.8(8.7-12.7)^{\star} \dagger$ & $<0.001$ \\
\hline$\% \mathrm{CV}$ & $15.4(13.0-18.6)$ & $22.3(18.9-26.5)^{\star}$ & $26.1(19.7-32.4)^{*} \dagger$ & $27.4(20.8-37.7)^{*} \dagger$ & $<0.001$ \\
\hline
\end{tabular}

Data are median $\left(25^{\text {th }}-75^{\text {th }}\right.$ percentile) values or numbers $n$. The groups were compared using Analysis of Variance (ANOVA), the Kruskal-Wallis one way analysis and Holm-Sidak or Dunn's test, where appropriate. ${ }^{*} P<0.05$ vs. $\leq 5.9$; ${ }^{\dagger} P<0.05$ vs. the two lowest categories.

Table 2: Correlation matrix presented as Spearman correlation coefficients $r$ for CGM profile complexity defined by the multiscale entropy index and quality indices of glucose control.

\begin{tabular}{|c|c|c|c|c|c|c|c|c|c|}
\hline & MSE index & Q-score & ADRR & LBGI & HGBI & GRADE & $\%$ GRADE $_{\text {hypo }}$ & $\%$ GRADE $_{\text {eu }}$ & $\%$ GRADE $_{\text {hyper }}$ \\
\hline MSE index & 1 & & & & & & & & \\
\hline Q-score & -0.353 & 1 & & & & & & & \\
\hline ADRR & -0.337 & 0.959 & 1 & & & & & & \\
\hline LBGI & -0.131 & -0.11 & -0.152 & 1 & & & & & \\
\hline HBGI & -0.292 & 0.905 & 0.901 & -0.450 & 1 & & & & \\
\hline GRADE & -0.286 & 0.911 & 0.918 & -0.430 & 0.969 & 1 & & & \\
\hline$\%$ GRADE $_{\text {hypo }}$ & -0.08 & 0.08 & 0.06 & 0.855 & -0.265 & -0.223 & 1 & & \\
\hline$\%$ GRADE $_{\text {eu }}$ & -0.308 & -0.938 & 0.952 & 0.306 & -0.957 & -0.960 & 0.110 & 1 & \\
\hline$\%$ GRADE $_{\text {eu }}$ & -0.308 & 0.814 & 0.830 & -0.541 & 0.960 & 0.917 & -0.377 & -0.929 & 1 \\
\hline
\end{tabular}

Values significant at the $p<0.001$ level (two sided) are shown in bold font. 

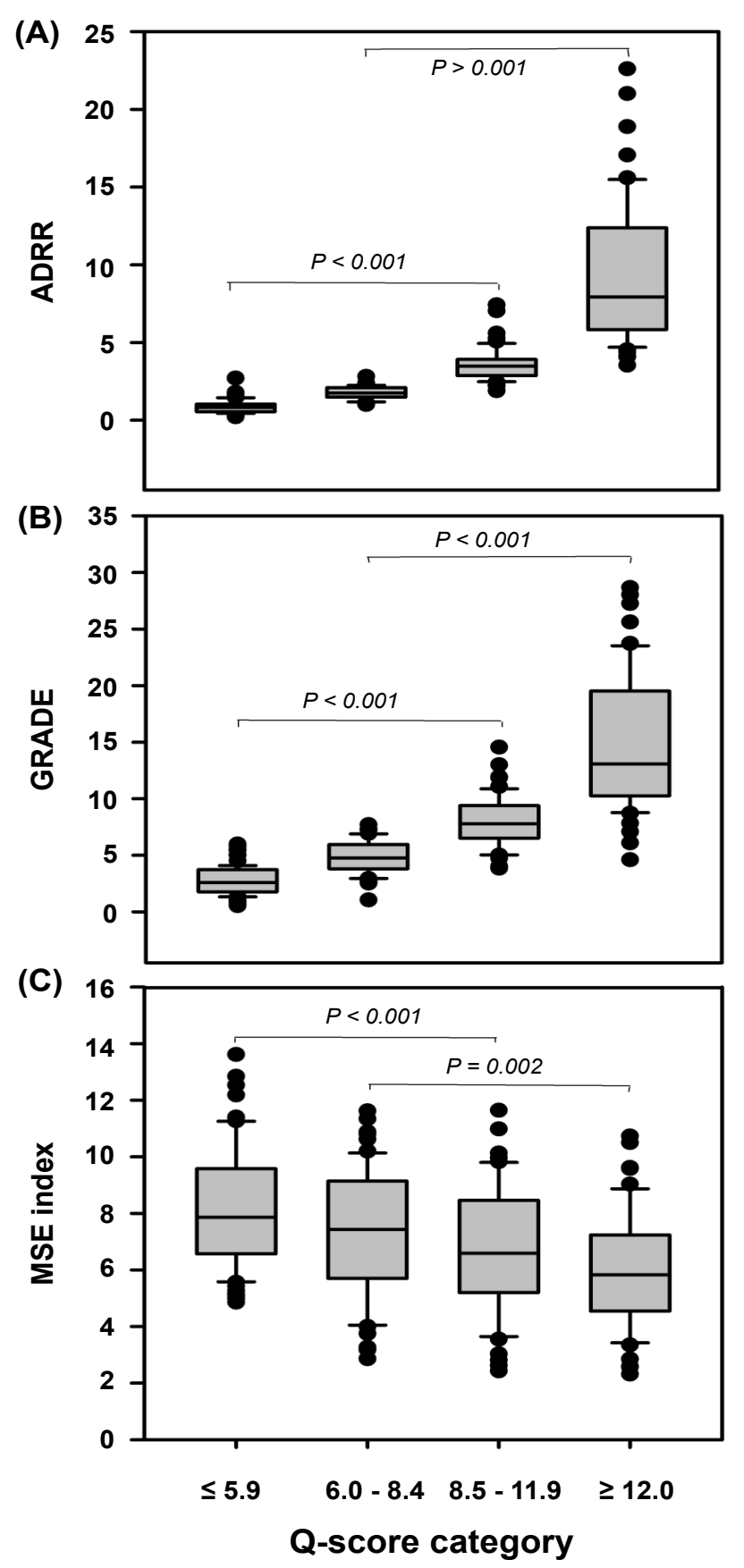

Figure 1: Differentiation between quality of glycemic control based on $\mathrm{Q}$-score categorization in the study cohort of nondiabetic $(n=12)$, T2D $(n=222)$, and T1D $(n=22)$ subjects using the ADRR (Average Daily Risk Range), GRADE (Glycemic Risk Assessment Diabetes Equation) score, and the Multiscale Entropy (MSE) index. Betweencategory differences were estimated by one-way analysis of variance and were statistically significant $(p<0.001)$. Q-score categories and quality of glycemia: $\leq 5.9$; Good, 6.0-8.4; Satisfactory, 8.5-11.9; Fair and $\geq 12.0$; Poor.

We found weak to moderate and inverse correlations between the previous indices and the MSE index, but slightly stronger ones between the Q-score and the MSE $(r=-0.353, p<0.001)$ than with ADRR or GRADE. A curvilinear relationship existed between MSE and the Q-score (not shown) according to the formula: $y=$ $14.274-0.910+0.016 x^{2}$.

Thus, an increase in the Q-score, that is, deteriora- tion of diabetes control is associated with a nonlinear decrease in the MSE index values.

The mode of the therapy affected moderately the indices of quality of glycemic control as disclosed by similar correlation coefficients of ADRR, GRADE, and Q-score with the anti diabetes treatment $(r=0.424, p<0.001$; $0.428, p<0.001$; and $0.472, p<0.001$, respectively). We used stepwise forward regression analysis to assess the independent effects of therapy and MSE as well as possible confounders on the Q-score. We included anti diabetes treatment, MSE, diabetes duration, age, sex, and body mass index. This analysis verified that the anti-diabetic therapy and MSE were predictor variables associated with the $Q$-score $(B=1.678 \pm 0.274, p<0.001$; MSE, $\beta=-0.364 \pm 0.115, p=0.002$, with therapy alone $R^{2}=0.201$, with both $R^{2}=0.233$ ). Diabetes duration and other confounders such as age, sex, and body mass index failed to contribute significantly. ADRR and GRADE, as dependent variables, produced lower $R^{2}$-values than the $\mathrm{Q}$-score in this regression model (not shown).

We further observed weak, inverse correlations between the MSE complexity index and measures of glucose exposure: $\mathrm{HbA1c}(r=-0.222, p<0.001)$ and mean glucose $(r=-0.177, p=0.004)$. The correlations of ADRR, GRADE, and Q-score with HbA1c ( $r=0.496$ to 0.576 ) and mean glucose ( $r=0.742$ to 0.901 ) were positive (all $p<0.001$ ) and more powerful than with the MSE index.

\section{Discussion}

We have shown in this retrospective cohort study that the indices of quality of glycemic control are composite measures of glycemia that can facilitate interpretation of CGM measurements in individual patients. This suggestion holds true especially for patients whose HbA1c levels are still in an acceptable range. Overall, ADRR, GRADE, and the Q-score are comparable as regards the capability of assessing the quality of glycemic control. In addition to ADRR and GRADE, the Q-score integrates, beyond the risk of hypo- and hyperglycemia, mean glucose, glucose range, and Mean of Daily Differences (MODD). The Q-score may thus be more suitable for therapeutic decision making than other items. Whether this score is superior to ADRR or GRADE needs further studies to be performed; however, this was not subject to this retrospective study. The analysis of glucose profile dynamics, as measured by the MSE index, extends the characterization of glucose profiles by providing insight into the dynamics of glucose regulation under different glycemic conditions. In this regard, the dynamical aspect gains increasing clinical relevance in diabetes $[13,14]$. The present results further show that numerically high-quality indices and a low MSE index characterize patients with insufficient glycemic control that requires therapy readjustment. Of note, we found a weak, negative relationship between glucose dynami- 
CS, as defined by the MSE index, and indices of the quality of glycemic control. Since the MSE index is a measure of system complexity, it can distinguish glucose time series of different complexity but cannot serve on its own as metric of short-term glycemic control. Quality indices are preferable to conventional glucose metrics [15], because they combine several glycemic parameters, for example, hypo- and hyperglycemic events, and glycemic variability. And the Q-score, in addition to these parameters, integrates multiple factors of glycemic control and may thus be the preferred index for the daily judgment of glycemia. However, ADRR, GRADE, and the Q-score are lesser sensitive to hypoglycemic than to hyperglycemic events. This is at least unexpected with respect to the Q-score in as much as the formula for its computation gives equal weight to hypo- and hyperglycemic events. A most likely explanation for this observation is the relatively small number of hypoglycemic events among the study cohort. Nevertheless, the sensitivity of the Q-score to hypoglycemic events needs clarification in subsequent studies. HbA1c and mean glucose measures of glucose exposure do not reflect the full picture of diabetes control because they do not account for glycemic variability [16] or changes in glucose dynamics [14] and are thus less suitable for assessment of glucose profiles from CGMS measurements and adjustment of patient-centered diabetes therapy.

Although the indices of the quality of glycemic control increased concomitantly with the loss of regularity in the CGM time series, the correlation between glucose complexity and quality of glycemia was weak. The unaccented association between quality of glycemic control and MSE index was unexpected; however, this might be explained by yet unknown metabolic factors involved in glucose regulation and not reflected in measures of overall glycemia.

Weaknesses of the current investigation are its retrospective nature and the relatively small number of T1D and ND subjects contained in the cohort. The strength is the inclusion of patients with T1D as well as T2D with different anti-diabetic treatments, including insulin and various classes of non-insulin anti-diabetic drugs.

In summary, the indices of quality of glucose control complemented with the dynamical complexity index described herein enable the comprehensive assessment of continuous glucose monitoring data and stratification of the glycemic status of patients with various therapy modalities. The judgment of glycemic control regarding Good, Satisfactory, Fair, and Poor should simplify interpretation of CGM outcomes.

\section{Financial Disclosures}

The authors declare that they have no relevant financial relationships to disclose.

\section{Conflict of Interest}

The authors have no conflict of interest to disclose.

\section{Funding/Support}

No funding or any financial support has been received during this study.

\section{References}

1. The Diabetes Control and Complications Trial Research Group (1993) The effect of intensive treatment of diabetes on the development and progression of long-term complications in insulin-dependent diabetes mellitus. $\mathrm{N}$ Engl $\mathrm{J}$ Med 329: 977-986.

2. Rodbard D (2009) Interpretation of continuous glucose monitoring data: Glycemic variability and quality of glycemic control. Diabetes Technol Ther 1: 55-68.

3. Kovatchev BP, Otto E, Cox D, Gonder-Frederick L, Clarke W (2006) Evaluation of a new measure of blood glucose variability in diabetes. Diabetes Care 29: 2433-2438.

4. Hill NR, Hindmarsh PC, Stevens RJ, Stratton IM, Levy JC, et al. (2007) A method for assessing quality of control from glucose profiles. Diabet Med 24: 753-758.

5. Thomas A, Schonauer M, Achermann F, Schnell O, Hanefeld M, et al. (2009) The "Glucose Pentagon": Assessing glycemic control of patients with diabetes mellitus by a model integrating different parameters from glucose profiles. Diabetes Technol Ther 11: 399-409.

6. Rodbard D (2009) Display of glucose distributions by date, time of day, and day of week: New and improved methods. J Diabetes Sci Technol 3: 1388-1394.

7. Rodbard D (2011) Clinical interpretation of indices of quality of glycemic control and glycemic variability. Postgrad Med 123: $107-118$.

8. Augstein $P$, Heinke $P$, Vogt $L$, Vogt $R$, Rackow $C$, et al. (2015) Q-score: development of a new metric for continuous glucose monitoring that enables stratification of antihyperglycemic therapies. BMC Endocr Disord 15: 22.

9. Costa M, Goldberger AL, Peng CK (2002) Multiscale entropy analysis of complex physiologic time series. Phys Rev Lett 89: 068102.

10. Augstein P, Vogt L, Kohnert KD, Heinke P, Salzsieder E (2010) Translation of personalized decision support into routine diabetes care. J Diabetes Sci Technol 4: 1532-1539.

11. Chen JL, Chen PF, Wang HM (2014) Decreased complexity of glucose dynamics in diabetes: evidence from multiscale entropy analysis of continuous glucose monitoring system data. Am J Physiol Regul Integr Comp Physiol 307: 179-183.

12. Costa MD, Henriques T, Munshi MN, Segal AR, Goldberger AL (2014) Dynamical glucometry: use of multiscale entropy analysis in diabetes. Chaos 24: 033139.

13. Fabris C, Sparacino G, Sejling AS, Goljahani A, Duun-Henriksen J, et al. (2014) Hypoglycemia-related Electroencephalogram changes assessed by multiscal entropy. Diabetes Technol Ther 16: 688-694.

14. Gough DA, Kreutz-Delgado K, Bremer TM (2003) Frequency characterization of blood glucose dynamics. Ann Biomed Eng 31: 91-97.

15. Patton SR, Clements MA (2013) Average daily risk range as a measure for clinical research and routine care. J Diabetes Sci Technol 7: 1370-1375.

16. Rodbard D, Baily T, Jovanovic L, Zisser H, Kaplan R, et al. (2009) Improved quality of glycemic control and reduced glycemic variability with use of continuous glucose monitoring. Diabetes Technol Ther 11: 717-723. 